Thomas Waitz

\title{
Verkehrsteilnehmer.
}

\section{Ein filmisches Versprechen, 1927/28}

DOI

https://doi.org/10.25365/phaidra.29

Original

Waitz, Thomas: Verkehrsteilnehmer. Ein filmisches Versprechen, 1927/28. In: Christoph Neubert/Gabriele Schabacher: Verkehrsgeschichte und Kulturwissenschaft. Analysen an der Schnittstelle von Technik, Kultur und Medien. Biel efeld: Transcript 2010.

Kontakt

t.waitz@univie.ac.at

Hinweis

Diese Textfassung weicht in geringfügigen Details von der Druckfassung ab.

Thomas Waitz

http://www.thomaswaitz.at/

ORCID https://orcid.org/0000-0002-0137-515X

\section{@creative}

Dieses Werk ist unter Creative Commons Namensnennung - Keine kommerzielle Nutzung Keine Bearbeitungen 4.0 International (CC BY-NC-ND 4.0) lizenziert. Um die Lizenz anzusehen, gehen Siebitte zu: https://creativecommons.org/licenses/by-nc-nd/4.0/legalcode.de 


\section{VERKEHRSTEILNEHMER. EIN FILMISCHES VERSPRECHEN, 1927/28 \\ THOMAS WAITZ}

\section{Verkehr}

Die Einstellung eines Films, 1927: Das Bild zeigt eine Pferdekutsche, auf deren zur Seite hin offener Ladefläche ein Sarg transportiert wird. Der Wagen ist reich verziert: Intarsien schmücken die Konstruktion, Kränze und Schleifen bedecken den schweren Holzsarg. Es ist eine kurze Einstellung, der dieses Bild entstammt, und sie ist eingewoben in ein Geflecht anderer, disparater Motive: Menschen, die umhergehen, Straßenbahnen, die vorbeifahren, Autos, unablässige Verkehrsströme. Das Bild des Leichenwagens fällt in diesem Fluss kaum auf, es ist mittels der filmischen Montage integriert in die Gleichzeitigkeit von Signalen, Zeichen und Bewegungslinien. Die Profanität des alltäglichen, nicht abschließenden, funktionalistischen Geschehens auf der Straße und die sakrale Intimität des bürgerlichen Trauerrituals zwischen Entblößung und Erbarmen, die noch von Stillstand, von einem Ende, von Transzendenz kündet hier treffen sie aufeinander. Und doch liegt in diesem Zusammenprall keine agonale Spannung, allenfalls ein Moment verhaltener Kuriosität. Die Sequenz, der diese Einstellung wiederum entnommen ist, findet sich in Walter Ruttmanns BerLIN. Die Sinfonie DER GROßSTADT (1927). Ruttmanns Querschnittsfilm nimmt sie immer wieder in den Blick: Bilder des Verkehrs in der Großstadt Berlin.

Als kulturelles Artefakt verweist die Kutsche, die den Sarg transportiert, auf die äußeren Repräsentationsformen eines vormodernen Rituals. Doch sie ist nicht nur Zeichenträger, sie ist auch Fahrzeug, eines unter vielen zudem. Die Expressivität des Schmuckes und seine demonstrative Außenwendung lässt dem Transport des Sarges Zeichencharakter zukommen. Doch wenn damit auf die körperliche Substanz eines bürgerlichen Individualitätskonzeptes, ja, auf die Kreatürlichkeit des Menschen selbst verwiesen wird, gefriert die Geste zur bloßen Behauptung: Der räumliche Kontext des Verkehrs (der einen primär filmischen Kontext darstellt) verweigert sich jeder Substanz; »die Devise der Neuen Sachlichkeit >Statt Ausdruck - Signale, statt Substanz - Bewegung > findet im Verkehr Spielraum und Milieu. « ${ }^{1}$ Der Film destrukturiert die vorfilmischen Inszenierungen: Suchende Kamerabewegungen, das Durchschneiden der Bildachse - eine Straßenbahn fährt vorbei - und ein jump cut

1 Helmut Lethen: Verhaltenslehren der Kälte. Lebensversuche zwischen den Kriegen, Frankfurt/Main: Suhrkamp 1994, S. 46. 
dezentrieren das Bildobjekt, wodurch die doppelte Rahmung des Sarges durch die Aufbauten der Kutsche und die Kadrierung des Bildes durchbrochen werden. Der damit verbundene Effekt wird durch die sich anschließende Montage noch verstärkt: Schon folgt der nächste Schnitt, weitere Straßenbahnen fahren vorüber, wir sehen das Gedränge auf einer großen Kreuzung, einen Verkehrspolizisten, der Anweisungen erteilt. Was der Film imaginiert, ist ein - so scheint es - fließendes, niemals zu einem Stillstand findendes Außen des Verkehrs; ein Außen, das vom Tod des empirischen Menschen nicht einmal mehr Notiz nimmt.

Einstellungen sich bewegender Droschken, Pferdewagen, Straßenbahnen, Autos und Passanten dienen Ruttmann zum einen als strukturierende Zwischenschnitte. Sie bilden aber in ihrer Sichtbarmachung, die über eine je narrative Funktion hinausweist, auch eine konkrete Evidenz der Erfahrung des Großstädtischen, das in einem spezifischen Verhältnis zum Medium Film steht.

Zum einen ist es der Film, der großstädtische Massen in einer Weise formiert, die der Erfahrung einer Massengesellschaft vorausliegt - und dies schließt die `Erfahrbarkeitく der Massenmobilisierung (der zivilen wie, wenig später, der militärischen) ein. Zum anderen ist die Wahrnehmung des Urbanen durch die Erfahrung des Films beeinflusst worden erinnert sei nur an das ästhetische Programm des Flanierens. ${ }^{2}$ Indem der Film schließlich mittels seiner medialen Apparatur als »technischem Äquivalent zum indifferenten Wahrnehmungsorgan des Großstädters « ${ }^{3}$ einer Wirklichkeit Gestalt verleiht und indem er als Massenmedium selbst Teil jener abstrahierten Form des Städtischen ist, welche sich »im Netzwerk ihrer Kommunikationssysteme gleichsam verdoppelt hat «, ${ }^{4}$ bilden die Erfahrung von Urbanität und das Dispositiv Kino strukturelle Zusammenhänge aus.

Doch was macht das Bild eines Leichenwagens, der sich im Verkehr befindet, aus dem historischen Nachhinein so bemerkenswert? Für was mag dieses Bild stehen - nicht im Sinne einer filmischen Rhetorik, son-

2 Vgl. Anne Friedberg: Window Shopping. Cinema and the Postmodern, Berkeley: University of California Press 1994.

3 Lothar Müller: »Die Großstadt als Ort der Moderne«, in: Klaus R. Scherpe (Hg.), Die Unwirklichkeit der Städte. Großstadtdarstellungen zwischen Moderne und Postmoderne, Reinbek: Rowohlt 1988, S. 14-33, hier S. 14

4 Heinz Ickstadt: »Kommunikationsmüll und Sprachcollage. Die Stadt in der amerikanischen Fiktion der Postmoderne «, in: Scherpe (Hg.), Die Unwirklichkeit der Städte, S. 197. 
dern im Hinblick auf etwas, das sich seine »semantische Substanz $\aleph^{5}$ nennen ließe? An welche Diskurse lässt sich dieses Bild rückbinden?

Die Wahl des Motivkomplexes und die Inszenierungsstrategien, derer sich Ruttmann bedient, sind kennzeichnend, weil sich die kulturelle Praxis des großstädtischen Verkehrs substanziell an die der Moderne innewohnende Erfahrung einer Dezentrierung und Auflösung des Subjekts koppelt. Das Medium Film ist es, das 1927/28 nicht nur ein solches Problem formuliert, sondern das zugleich von einem Versprechen kündet: dem Versprechen des Verkehrs.

Das Zeitalter der modernen, individuellen Massenmobilität beginnt erst um 1960 und ist damit identisch mit der Verfügbarkeit des Automobils für breite Teile der Bevölkerung. Doch eine entscheidende Popularisierung und Wegbereitung von Mobilität zeichnet sich bereits in den 1920er Jahren ab. So ist es die Eisenbahn, die eine neue Sozialstruktur von ländlichem Wohnen und städtischem Arbeiten ermöglicht. 1926 verbinden sich zahlreiche kleine Fluggesellschaften zur deutschen Lufthansa AG, 1930 ist der erste Direktflug zwischen den Kontinenten möglich. Das Automobil wandelt sich von einem Luxusgut, das der gesellschaftlichen Repräsentation und dem Vergnügen dient, zu einem Mittel der Raumüberbrückung. Zwar können sich nur die wenigsten tatsächlich ein eigenes Auto leisten. Es steht jedoch - etwa durch den Autorennsport bereits in den 1920er Jahren "ganz zentral im Erwartungshorizont der Menschen «. ${ }^{6}$ Bei all diesen Entwicklungen ist nicht einmal entscheidend, ob sich die mit der zunehmenden Mobilität verbundenen Erwartungen auch tatsächlich realisierten. Entscheidend ist allein ihre Behauptung nur so stieg etwa das Auto »zum Symbol der Freiheit und Unabhängigkeit « ${ }^{7}$ auf.

Gleichzeitig mit der Ausbildung eines Zeitalters der Mobilität bilden die 1920er Jahre einen Zeitraum nachhaltiger Diffusion und sozialer Desorganisation, in deren Verlauf die stabilen Außenhalte der Konventionen, vertraute Ordnungen und die Gehäuse der Tradition brüchig werden. Auf der einen Seite stehen eine weitverbreitete großstadtfeindliche Grundhaltung und agrarromantische Stimmungen, die Entlastung von den problematischen Verwerfungen der modernen Gegenwart versprechen. Auf der anderen Seite behauptet eine avantgardistische Anthropo-

5 Christian Metz: »Bemerkungen zu einer Phänomenologie des Narrativen«, in: ders., Semiologie des Films, München: Fink 1972, S. 38.

6 Rainer Gömmel: »Technik und Mobilität«, in: Armin Hermann/Wilhelm Dettmering (Hg.), Technik und Gesellschaft, Düsseldorf: Georg-AgriculaGesellschaft 1993, S. 293-313, hier S. 308.

7 Ebd., S. 310. 
logie kaum weniger in ihren Absichten zu führen als den Versuch, ein Überwintern des Subjekts in der Moderne zu sichern - oder, um das Bild aufzugreifen, das sie sich selbst erwählt: Die Reintegration des Menschen in eine hochgradig dissoziierte, moderne Gesellschaft im Geiste seiner Adressierung als Verkehrsteilnehmer.

Derjenige, der die Bedingungen, Notwendigkeiten und Chancen eines solchen Menschenbildes skizziert, ist Helmuth Plessner. In einer historisch-politischen Situation, in der die Gewissheiten einer überkommenen Gesellschaftsstruktur einstürzen, verleiht Plessner in seiner 1924 erschienenen Schrift Grenzen der Gemeinschaft einer Anthropologie Kontur, die Aufenthaltslosigkeit, Beweglichkeit und Distanz zur Maxime menschlicher Existenz erhebt.

In einer Zeit der Verunklarung >verbindlicher< Werte und normativer Entscheidungskriterien, einer Zeit der »geistig-politischen Rückläufigkeit des Bürgertums $\ll,{ }^{8}$ gelte es, einen Habitus zu privilegieren, der durch Merkmale wie »Wahrnehmungsschärfe, Nüchternheit und Realismus« oder, wie Helmut Lethen in seiner gleichermaßen wirkmächtigen wie folgenschweren Plessner-Lektüre vorgeschlagen hat, »Kälte« gekennzeichnet ist. $^{9}$

Plessners Schrift lässt sich in einem historischen Diskurs verorten, innerhalb dessen Gemeinschaft und Gesellschaft antagonistische Kategorien bilden. ${ }^{10}$ In seiner Besprechung der plessnerschen Kritik des sozialen Radikalismus, so das Werk in seinem Untertitel, schreibt Siegfried Kracauer:

»Versteht man unter dieser etwa das anorganische Getriebe der entseelten, nur noch zweckbestimmten Menschen, die sich in der durch Kapitalismus und Technik mechanisierten Welt bewegen, so wird jene als das organische Miteinander der ganzen Menschen gedacht, die sich in die richtige Ordnung zu schicken wissen. $\ll^{11}$

8 Helmuth Plessner: Grenzen der Gemeinschaft. Eine Kritik des sozialen Radikalismus, Frankfurt/Main: Suhrkamp 2002, S. 44.

9 Lethen: Verhaltenslehren der Kälte.

10 Vgl. Rolf Parr: »)Gemeinschaft $\prec$ und `Gesellschaft $\prec$. Diskursive Koordinaten im wilhelminischen Deutschland«, in: ders., Interdiskursive As-Sociation. Studien zu literarisch-kulturellen Gruppierungen zwischen Vormärz und Weimarer Republik, Tübingen: Niemeyer 2000, S. 47-54.

11 Plessner: Grenzen der Gemeinschaft, S. 268. 
Die >mechanisierte Künstlichkeit der gesellschaftlichen Verhältnisse hat Helmuth Plessner jedoch nicht zu überwinden getrachtet, sondern in ihr gerade die Aussicht auf einen glückenden Selbstentwurf des Individuums gesehen. Und deshalb begrüßt er »traditionell negativ bewertete Merkmale wie Anonymität, Aufenthaltslosigkeit, Zerstreuung und Seinsentlastung [...] als Möglichkeitshorizont [...], ohne den sich eine menschliche Existenz nicht auf spezifisch humane Weise verwirklichen kann. « $^{12}$

Helmut Lethen hat zu zeigen versucht, wie die Literatur der Neuen Sachlichkeit den von Plessner initiierten Diskurs aufgreift. Den Schriften von Jünger, Schmitt, Brecht und anderen schreibt er den Charakter von »Anweisungen « zu und spricht von »Verhaltenslehren der Kälte«. Ihre Funktion liege darin, »Eigenes und Fremdes, Innen und Außen [zu] unterscheiden « ${ }^{13} \mathrm{Ihr}$ implizites Ziel sei die Gewinnung von Verhaltenssicherheit in der Erlernung von Techniken, »mit denen sich die Menschen nahe kommen, ohne sich zu treffen, mit denen sie sich voneinander entfernen, ohne sich durch Gleichgültigkeit zu verletzen «. ${ }^{14}$

Im symbolischen Zaubermittel der »kalten persona $«{ }^{15}$ die ihre Identität in der Fremdwahrnehmung der wetteifernden Individuen bestimmt, findet sie ihre Gestalt. `Distanz $`$ - so Plessners These - werde im Angesicht von Modernisierungsschüben zur Überlebensstrategie des Subjekts. ${ }^{16}$ In der Folge sieht Lethen die zentrale Bedeutung des plessnerschen Werkes darin, »dem Menschen einen angstfreien Zugang zum Prozeß der Modernisierung zu erschließen und einen Freiheitsspielraum zu konstruieren $\ll{ }^{17}$

»Doch da diese Mitwelt >immer unversöhnlich ist, wirft ihr Spiegel dem Subjekt ein Bild realitätstüchtiger Selbsterkenntnis zu; denn es geht ums Überleben. Nur gespannte Wachsamkeit und die Bereitschaft, sich jederzeit aus Bindungen zu lösen, gewährleisten Mobilität. «18

12 Lethen: Verhaltenslehren der Kälte, S. 8.

13 Ebd., S. 7.

14 Plessner: Grenzen der Gemeinschaft, S. 80.

15 Lethen: Verhaltenslehren der Kälte, S. 53.

16 Vgl. Christoph Asendorf: Entgrenzung und Allgegenwart. Die Moderne und das Problem der Distanz, München: Fink 2005.

17 Lethen: Verhaltenslehren der Kälte, S. 43.

18 Ebd., S. 58. 
Die Mobilität aber, von der hier die Rede ist, ist jene des Verkehrs. Und obwohl der Begriff noch am Anfang des 20. Jahrhunderts weniger den späteren Straßenverkehr meint als - im Sinne Norbert Elias' ${ }^{\prime 9}$ - die $» G e-$ samtheit der Beziehungen der Menschen untereinander «, wie es der Große Brockhaus des Jahres 1911 verzeichnet, geschieht die Wahl dieser Vokabel höchst bewusst. »Gesellschaft ohne Technik und Zivilisation ist nicht möglich, Technik im weitesten Sinne genommen«, heißt es bei Plessner. Und weiter: »Jeder Verkehr zwischen Menschen, welcher des Werkzeugs, des künstlichen Mittels bedarf, hebt sich aus der Gemeinschaftssphäre heraus. $\ll^{20}$ Es braucht also nicht viel, um in der $»$ kalten persona« Plessners die Konturen einer Figur zu erkennen, die in ihren prototypischen Eigenschaften "gespannte[r] Wachsamkeit« v.a. eins zu sein scheint: ein Verkehrsteilnehmer.

Mit der Neuen Sachlichkeit wird der Verkehr zu einem wesentlichen Topos - etwa in der Literatur, und das nicht nur im Hinblick auf die »Asphalt «- und Großstadtliteratur. ${ }^{21}$ Auch im Kino findet der Verkehr ein Feld der Repräsentation, etwa im Genre des Straßenfilms, das von der verbreiteten Angst vor dem sozialen Abstieg kündet. Doch entscheidend für die Bedeutung des Verkehrs ist nicht seine motivische Dimension, sondern die Tatsache, dass er in einem umfassenderen Sinne als Wahrnehmungsmodell figuriert.

1930 berichtet Siegfried Kracauer in einem bemerkenswerten Aufsatz, der den beziehungsreichen Titel »Kleine Signale« trägt, von zweierlei: dem Besuch eines Caféhauses und dem Phänomen des gelben Lichts der Verkehrsampeln. Beides scheint wenig miteinander zu tun zu haben. Und doch erkennt Kracauer eine Verbindung: Sie liegt für ihn darin, wie der Einzelne »entmenschlicht« werde, der Caféhauskellner wie der Autofahrer. »Es fehlt der menschliche Kontakt zwischen den Menschen, sie sind nur noch die Vollstrecker ökonomischer und sozialer Funktionen. Apparate und Idole stehen einander hart gegenüber. $\ll^{22}$

So sei der Kellner nicht aufgrund eines Selbstverständnisses freundlich zum Gast, sondern weil er dazu ermahnt worden sei. In der gleichen Weise wirke die Gelbphase der Verkehrsampeln, denn »durch die Ein-

19 Vgl. Norbert Elias: Über den Prozeß der Zivilisation, Frankfurt/Main: Suhrkamp 2001, S. 12.

20 Plessner: Grenzen der Gemeinschaft, S. 40.

21 Vgl. Johannes Roskothen: Verkehr. Zu einer poetischen Theorie der Moderne, München: Fink 2003.

22 Siegfried Kracauer: »Kleine Signale«, in: ders., Schriften. Bd. V.2: Aufsätze 1927-1931, Frankfurt/Main: Suhrkamp 1990, S. 234-236, hier S. 235. 
schaltung des Zwischenlichts wird die Rücksichtnahme gewissermaßen objektiviert und die Initiative aus den Menschen herausgesetz $\ll .23$

Bezeichnend an diesen Äußerungen scheint nicht so sehr die für Kracauer durchaus typische Melancholie zu sein, die ihn schließen lässt: »Ich wünschte, daß auch bei uns das gelbe Licht draußen erlöschte und in die Menschen zurückkehrte«; es ist die Beiläufigkeit, mit der Verkehr als Wahrnehmungsmodell dafür dient, wie Gesellschaft >funktioniert « denn in dieser Rhetorik liegt eine Grundsätzlichkeit, die stärker ist als jede - so konkrete wie sentimentale - Kritik an Usancen des Gastgewerbes, welche sich oberflächlich lesen ließe.

Der Zusammenhang zwischen solch sprachlichen Bildern des Verkehrs und sozialen Entwicklungen aber ist struktureller Natur. Ernst Jünger beobachtet zur selben Zeit: »Es sind große Bezirke, wo man in steigendem Maß durch Schwingung und Reflex zu handeln beginnt; das gilt im besonderen für den Verkehr. « ${ }^{24}$ Und wenn Helmuth Plessner 1924 die Vorteile des Lebens in der »Gesellschaft« gegen die Stickluft des Gemeinschaftskultes abhebt, beschreibt er das Medium der Existenz als »offenes System des Verkehrs zwischen unverbundenen Menschen «. ${ }^{25}$ Diese öffentliche Sphäre wird von ihm gerade deshalb begrüßt, weil sie frei sei von fundamentalistischen Werten:

»In jeder Sphäre des Verkehrssystems muß dem Gedanken der restlos realisierbaren Ordnung die Gesetzlichkeit des reinen Wertes geopfert werden, denn das Medium, welches sein lauteres Licht ablenkt und trübt, ist die unüberwindliche Unverbundenheit des Menschen im Daseinsgebiet zwischen Familiarität und Objektivität. $\ll^{26}$

Diese Gegensätze, die Plessner anführt - »Familiarität und Objektivität« -, bilden jenes Spannungsfeld aus, das filmisch dort evident wird, wo eine Kutsche, die einen Sarg transportiert, mittels filmischer Montage in den Verkehr gerät. In diesem Sinne ist die Kuriosität, als die das Bild des Sargtransportes gelesen werden kann, das Ergebnis einer Latenz. Wenn gilt, dass der Effekt des Komischen stets auf eine Konstellation der Ungleichzeitigkeit rückführbar ist, dann liegt diese im ruttmannschen Beispiel in der Diskrepanz, welche sich ergibt aus dem Bedeutungskern des

23 Ebd.

24 Ernst Jünger: Das abenteuerliche Herz. Figuren und Capriccios, Stuttgart: Klett Cotta 1979, S. 173.

25 Plessner: Grenzen der Gemeinschaft, S. 40.

26 Ebd., S. 95. 
bürgerlichen Trauerrituals, auf den noch selbst der periphere Transport eines Sarges verweist, und der Einbettung in den sich allein unter funktionalistischen Gesichtspunkten organisierenden Verkehr.

So schlussfolgert Siegfried Kracauer: »Der Verkehr ist heute mehr und mehr in sein Zeitalter gelangt. Alles verkehrt miteinander, jede Schranke ist aufgehoben . $^{27}$ Zugleich wird er zur Gewohnheit. »Die Macht, die er ausübt, übertrifft die großer Umstürze $«{ }^{28}$ merkt Kracauer ein anderes Mal lakonisch an. Es ist eine Macht, die - und das sind wiederum filmische Verhaltenslehren - zu ignorieren tödlich sein kann.

\section{Film}

Das Bild des Leichenwagens im Verkehr, welches aus Ruttmanns BerlinFilm stammt, findet sich ein weiteres Mal. Auch King Vidors Film THE CROWD (1928) enthält das Bild eines Sargtransportes. Auf eine fast ebenso beiläufige Art zeigt er einen Leichenwagen inmitten des Verkehrs. Betrachtet man die beiden Bilder nebeneinander, scheinen sie wie Zitate des jeweils anderen. Diese Koinzidenz mag über einigen anekdotischen Wert verfügen, aber daneben verweist sie - über die Grenzen zweier Kontinente, Gesellschaften und Filme - auf eine gemeinsame Wirklichkeitserfahrung am Ende der 1920er Jahre. Diese Erfahrung ist - darin liegt die Parallele zum Ruttmann-Film - das Bild einer Gesellschaft, die sich im Verkehr befindet. Auch in THE CROWD wird auf diesen tiefer liegenden Diskurs verwiesen, aber in semantischer Hinsicht erfährt das Paradigma eine entscheidende Verschärfung. In ihrem narrativen Kontext das soll im Folgenden gezeigt werden - versinnbildlicht die entsprechende Sequenz jene Bedrohung, welche sich aus einer Verweigerung gegenüber dem funktionalistischen Verkehr fast zwangsläufig ergeben muss, in einer Konsequenz, die das plessnersche »Überleben« zu deklinieren scheint.

Vordergründig erzählt THE CROWD aus dem Leben eines jungen Amerikaners, John Sims, der nach New York kommt, »one of 7 million who believe New York depends upon them«, wie ein Zwischentitel zu Beginn sarkastisch anmerkt - eine klassische Geschichte von den Schwierigkeiten des amerikanischen Traums also, jenem ideologisch ge-

27 Siegfried Kracauer: »Chauffeure grüßen«, in: ders., Schriften. Bd. V.1: Aufsätze 1915-1926, Frankfurt/Main: Suhrkamp 1990, S. 376-377, hier S. 377.

28 Siegfried Kracauer: »Stadt-Erscheinungen«, in: ders., Schriften. Bd. V.3: Aufsätze 1932-1965, Frankfurt/Main: Suhrkamp 1990, S. 90-93, hier S. 93. 
wendeten Versprechen des sozialen Aufstiegs im Geiste der protestantischen Ethik Max Webers. Verlobung, Heirat, die Geburt zweier Kinder die folgenden narrativen Stationen repräsentieren zwar die klassischen Elemente eines bürgerlichen Lebensentwurfes, aber der Film zeigt unverhohlen, dass die damit verbundenen Versprechen nur zum Teil eingelöst werden. Er schildert Johns wenig glanzvolles Arbeitsverhältnis, das wie ein Hohn auf das verinnerlichte Selbstbild desjenigen scheint, dem die große Zukunft noch an der Wiege versprochen wurde. John ist Angestellter in einem Großraumbüro - Dutzende von exakt auf imaginären Linien im Raum aufgereihten Schreibtischen markieren einen Raum, der in kleine Areale aufgeteilt ist, die den Nachbarfeldern ohne jeden Schutz und ohne Grenzen beigeordnet sind - eine Anordnung, welche die Anonymität des Einzelnen unmittelbar verdeutlicht. Das Büro symbolisiert daher nicht nur das Großkapital, ${ }^{29}$ es ist zugleich evident gewordene Sozialstruktur. Auch die beschränkte Wohn- und Finanzsituation erweist sich zunehmend als problematisch und überschattet immer stärker Johns Beziehung zu seiner Frau Mary. Der Unfalltod der Tochter läutet schließlich den sozialen Abstieg ein, der melodramatisch inszeniert wird. Vom Gedanken an das tote Kind auch bei der Arbeit verfolgt, kündigt John. In einer ganz auf dramatische Effekte hin inszenierten Szene droht er, sich von einer Eisenbahnüberführung zu stürzen; nur sein kleiner Sohn hält ihn ab.

Nun ist THE CROWD, »die Geschichte eines Menschen, der das Leben beobachtet $«{ }^{30}$ weit davon entfernt, eine unter zahlreichen zeitgenössischen Variationen einer Degradierungsfabel zu sein. Dass in Augenblicken sozialer Desorganisation der drohende Abstieg zum allverhandelten Topos wird, scheint überdies kaum überraschend - allein die Weimarer Republik hat ein ganzes Arsenal solcher Erzählungen hervorgebracht. Bereits zu Beginn von THE CROWD macht King Vidor jedoch deutlich: Hier wird nicht allein ein individuelles Schicksal verhandelt, hier werden Aussagen getroffen, die über die Schilderung eines Einzelfalls hinaus Geltung beanspruchen und die modellhaft die US-amerikanische Gesellschaft als - um mit Benedict Anderson zu sprechen - Projekt einer »Imagined Community « ${ }^{31}$ in den Blick nehmen. Dies verdeutlicht insbesonde-

29 Vgl. Jerzy Toeplitz: Geschichte des Films. Bd. 1: 1895-1928, Berlin: Henschel 1984, S. 413.

30 King Vidor: A Tree is a Tree. An Autobiography, Hollywood: Samuel French 1981, S. 102.

31 Benedict Anderson: Imagined Communities. Reflections of the Origin and Spread of Nationalism, London/New York: Verso 1983. 
re jene Sequenz, die der eigentlichen Handlung wie eine Präambel vorausgestellt ist.

Am 4. Juli 1900 kommt John Sims zur Welt, und damit zugleich im Jahre Null des neuen Jahrhunderts und am amerikanischen Unabhängigkeitstag, womit der symbolische Bezugsrahmen gespannt ist, den der Film einnehmen wird. Die folgende, in sinnbildlichen Ereignissen verdichtete Schilderung der Lebensumstände des kindlichen und juvenilen Protagonisten zeigt ein spezifisches soziales Milieu, das durch Prosperität, Standesbewusstsein, patriarchale Strukturen und damit eine spezifische Sozialisation geprägt ist, die der Soziologe David Riesman später als »innen-geleitet« beschreiben wird. In seiner Studie The lonely Crowd fasst er unter diesem Begriff einen Prozess, dem zufolge »die Kraft, die das Verhalten des Individuums steuert, verinnerlicht, d.h. frühzeitig durch die Eltern in das Kind eingepflanzt und auf prinzipiellere, aber dennoch unausweichliche Ziele gerichtet wird. « ${ }^{32}$

Gleich einem »seelischen Kreiselkompass «, ${ }^{33}$ so Riesman, habe dieser Typus die Steuerungskräfte seines individuellen Handelns mittels Sozialisation verinnerlicht. Im Falle John Sims heißt das so festgelegte, »unausweichliche Ziel«: »There's a little man the world is going to hear from«, wie der Vater unmittelbar nach der Geburt prophezeien wird. Zwölf Jahre später - umfallende Spielkarten symbolisieren die zeitliche Ellipse - ist es John selbst, der diese Einschätzung habitualisiert hat. In einer Gruppe von Kindern, die von ihren späteren Berufswünschen erzählen, - eine der wenigen Szenen, die eine innerdiegetische Identitätskonstruktion in Form einer Selbsterzählung darstellen - proklamiert er, die Einschätzung des Vaters antizipierend: »My Daddy says, I'm goin' to be somebody big!«

Damit erfährt John Sims eben jene »Einpflanzung« von naivem Selbstvertrauen und Überlegenheitsgefühl, in welcher der Film später, wenn der Held in die Metropole New York kommt, einen Großteil der Anpassungsschwierigkeiten an ein sozial grundlegend anders strukturiertes Leben innerhalb der großstädtischen Massengesellschaft sehen wird. Denn in Sims' späterem Scheitern manifestiert sich auch die Krise einer Klasse, die Siegfried Kracauer in Die Angestellten beschrieb: einerseits gefangen zwischen kleinbürgerlicher Prätention und Standesdünkeln der Arbeiterklasse gegenüber, andererseits verharrend in konformistischen Verhaltensweisen, in denen sich das Streben nach Distinktion

32 David Riesman: Die einsame Masse. Eine Untersuchung der Wandlung des amerikanischen Charakters, Reinbek bei Hamburg: Rowohlt 1958, S. 36.

33 Ebd., S. 32. 
ausdrückt. ${ }^{34}$ Am Ende der 1920er Jahre reagiert THE CROWD damit auf eine soziale Wirklichkeit, die in Weimar wie in den USA durch eine zunehmende Diskrepanz gekennzeichnet ist zwischen der fortgesetzten Behauptung eines bürgerlichen Identitätsentwurfes und gesellschaftlichen Entwicklungen, die von Serialität und Massenproduktion geprägt sind.

Die fortwährende Zunahme der Produktivkräfte, die ihrerseits mit einer sich stets beschleunigenden Reproduzierbarkeit einhergeht, ermöglicht zum Ende der 1920er Jahre des letzten Jahrhunderts nicht nur die Massenproduktion von Waren; sie zwingt auch die Produzenten, ihren Anspruch auf Individualität aufzugeben, der seit der Renaissance die grundlegende Ideologie des Bürgertums bildete. Und dies betrifft nicht nur die Arbeit am Fließband, sondern einen auf Dauer gestellten Prozess fortwährender sozialer Reorganisation.

Während jedoch die Verfasstheit der Weimarer Republik zutiefst von der ins Pathologische reichenden Erfahrung des verlorenen Weltkriegs geprägt ist, stehen die 1920er Jahre in den USA bis zum Beginn der Depression in der Kontinuität des Gilded Age der Jahrhundertwende, einer Zeit tief greifenden gesellschaftlichen Wandels und hoher Prosperität. V.a. der Konflikt zwischen einem älteren ländlichen und dem modernen städtischen Amerika ist es jedoch, in dem ein ideologischer Bruch zutage tritt. David Riesman hat beschrieben, wie sich im großen Rahmen ein Übergang von den herkömmlichen Verhaltensnormen einer auf Produktion, Erwerb, Sparsamkeit und Beständigkeit angelegten Gesellschaft zu denen eines "geltungssüchtigen Verbrauchs ${ }^{35}$ vollzog. Gemeinsam ist beiden, in wechselseitiger Beeinflussung stehenden Gesellschaften der USA und der Weimarer Republik - bei allen Unterschieden - die tiefe Spannung zwischen Tradition und Modernisierung, zwischen Fortschrittsgläubigkeit und Angst vor zu raschem Wandel, zwischen Urbanisierung und kleinstädtischer Nostalgie.

Eine Szene in THE CROWD spielt in ironischer Weise auf die Serialität der Massengesellschaft an: Nach Dienstschluss eilt John wie seine zahllosen Kollegen aus dem Großraumbüro in den Waschraum. Während er sein Gesicht - klassischer Ausdruck personaler Identität - im Spiegel betrachtet, wird er von seinen Kollegen im Vorübergehen geneckt. Ihre spöttischen Bemerkungen unterscheiden sich indessen kaum. »You birds have been working here so long that you all talk alike«, entfährt es John, halb erstaunt, halb verächtlich, denn er scheint damit auch deutlich zu machen: Ich bin nicht einer von euch, nicht einer wie alle.

34 Vgl. Siegfried Kracauer: Die Angestellten. Aus dem neuesten Deutschland, Frankfurt/Main: Suhrkamp 1971.

35 Riesman: Die einsame Masse, S. 299. 
Bezeichnenderweise bildet die `Dispositionssequenz $\prec$ zu Beginn auch in formaler Hinsicht einen scharfen Kontrast zum übrigen Film. Sie ist geprägt von einer expressionistischen Bildgestaltung, etwa in jener Einstellung, in der John Nachricht vom Tod des Vaters erhält und die über einen zentralperspektivisch orientierten, grafisch abstrahierten Bildaufbau verfügt: eine Treppe, die aufsteigend auf den Betrachter zuführt, am unteren Ende die amorphe Menge der restlichen Familienmitglieder, auf halber Höhe John, gestützt und zum Vorangehen aufgefordert von der Mutter, »half-way between his ruined past and an unknown future «. ${ }^{36}$

Die formale Gestaltung dieser Einstellung verweist auf zweierlei: in ihrer Auffälligkeit zum einen auf die Symbolträchtigkeit des Gezeigten als Initiationserlebnis und das Ende der so entscheidenden väterlichen Prägung für den »innen-geleiteten« Charakter. Zum anderen bildet die Zentralperspektive - historisch betrachtet - einen diskursiven Ausdruck. Denn sie stellt nicht nur eine Errungenschaft im Sinne >originaler (Wiedergabe von Wirklichkeit dar, sondern steht - gerade in der ersichtlichen Ausschließlichkeit, mit der sie in THE CROWD einmalig und an zweckhafter Stelle eingesetzt wird - auch für eine Entscheidung, den Zuschauer als betrachtendes Subjekt im Zentrum des Bildaufbaus zu positionieren; und dieser Standort des Subjekts bildet wiederum eine Verdoppelung der Position Johns auf der Ebene der Mise En Scène.

Wenn der weitere Verlauf des Films hingegen von einem sachlichen Stil und einer diversiver Wahrnehmung verpflichteten Bildgestaltung geprägt ist, vollzieht der Film in seinen stilistisch disparaten Teilen intratextuell eine doppelte Ablösung: vom expressionistischen Ausdruck hin zum Stil der Neuen Sachlichkeit, von der vormodernen Zentralperspektive zu vielfältigen, mehrfach gebrochenen und reflektierten Blickstrukturen - die insbesondere in der fragmentarisierenden Montage der Stadtansichten New Yorks zum Ausdruck kommen. Damit spiegelt THE CROWD mittels einer formal-ästhetischen Strategie bereits zu einem frühen Zeitpunkt eine Narration, die den Wandel korrespondierender Subjekterfahrungen als Folge der Krise der »Innen-Leitung«, zum Gegenstand nehmen wird.

Immer wieder zeigt THE CROWD die Figur des John Sims in Figurationen der Masse: am deutlichsten zu Beginn des Films in der geometrisch extrem stilisierten Totale auf das Heer der Büroangestellten, zum Schluss - gleichsam in Form einer narrativen Klammer - als Teil eines Vaudeville-Publikums, aber auch als einen der Wartenden vor dem Arbeitsamt, oder unter den zahlreichen, Vergnügen und Zerstreuung suchenden Gästen auf Coney Island. Die Bildinszenierungen mögen im Einzelnen unterschiedlich ausfallen, doch lässt sich in ihnen eine deutliche Entwick-

36 John Baxter: King Vidor, New York: Monarch 1976, S. 31. 
lung ablesen. Immer stärker wird dieser John Sims im Laufe des Films (der mit dem Verlauf seines Lebens korrespondiert) von der Menge absorbiert, immer stärker scheint er in dem ihn umgebenden, großstädtischen Verkehr aufzugehen. Den Wende- und Kristallisationspunkt dieser Inszenierungsstrategie bildet jene Szene, in der John sein totes Kind von der Fahrbahn aufhebt und auf seinen Armen, der ihn umschließenden Masse in umgekehrter Bewegungsrichtung entgegenschreitend, vom Unfallort fortträgt - ein Bild, das zu artikulieren scheint, was der Film einmal an anderer Stelle in einem kommentierenden Zwischentitel wie folgt benennt: »We do not know how big the crowd is and what opposition it is ... until we get out of step with it."

In dieser Aussage liegt der Schlüssel zu der hier vorgeschlagenen Lesart von THE CROWD, der zufolge der Film davon handelt, wie die Figur des John Sims künftig nicht mehr in >Opposition< zur Masse stehen wird, sondern ein Teil von ihr wird - und zwar, indem er seine Rolle als Verkehrsteilnehmer anzunehmen bereit ist. ${ }^{37}$ Doch noch ist es nicht so weit: In einer Szene, welche die Familie am Strand zeigt, singt John emphatisch: »All alone - I'm all alone«. In ironischer Absicht erfolgt ein plötzlicher Einstellungswechsel. Die Totale zeigt, dass von Einsamkeit nicht die Rede sein kann: Ein Gast beschwert sich über sein Musizieren, eine große Zahl weiterer teilt sich, dicht an dicht, den Platz an der Sonne - nur eine unter vielen Szenen, in denen individualistische Ideologie und Konformismus fordernde Massengesellschaft aufeinandertreffen, und zwar in einer Weise, die keine Versöhnung gestattet. Egoistisch wird John seine Ukulele nicht beiseitelegen und Mary mit der Zubereitung der Speisen an der Feuerstelle alleinlassen, und diese wird schließlich überfordert zusammenbrechen. »It's always the same«, klagt sie - worauf John nur zu entgegnen weiß: »Don't crab dear. Everything is goin' to be roses ... when my ship comes in.« - Eine Hoffnung, die sich freilich nicht erfüllen wird. Und schließlich ist selbst ein so privater Moment wie jener der Geburt des ersten Kindes, den der Film für eine komödiantische Eskapade zum Anlass nimmt, in der Sims' verzweifeltes Umherirren auf dem Krankenhausflur ausgestellt wird, in einer Weise visualisiert, die immer wieder die Effekte der Massengesellschaft in den Blick nimmt. Denn am Ende ist Sims wieder einmal nur einer unter vielen, als Wartender auf der Bank vor einem gewaltigen Kreissaal, und seine Frau nur eine unter zahllosen anderen Müttern, die mit ihr das Zimmer teilen. Wenn aber bereits die Geburt des Individuums nur noch über Serialität und

$37 »$ Masse « ist hier nicht in erster Linie als theoretischer Terminus zu sehen, wie ihn etwa Gustave Le Bon wenige Jahrzehnte zuvor eingeführt hatte, sondern als zunächst >naiver` Begriff, der die Erfahrungswelt der Großstadt in ihrem subjektiven Erleben erfassen soll. 
Massenförmigkeit erfahrbar ist, dann ist die bürgerliche Identität zur Gänze in der Krise angelangt.

Eine narrative Linie, in der sich King Vidors Film lesen lässt, sind das Scheitern eines bürgerlichen Lebensentwurfes des 19. Jahrhunderts und die Reintegration seines Protagonisten in eine massenkulturelle, auf Konsum gründende Gesellschaft der Moderne. ${ }^{38}$

Diese beiden, in der historischen Situation der ausgehenden 1920er Jahre auch in den USA noch durchaus konkurrierenden Entwürfe prallen aufeinander in einer Sequenz, die den Abschied der Protagonisten John und Mary von der zuvor - bemerkenswerterweise bei einem Verkehrsunfall - ums Leben gekommenen Tochter zeigt. Nicht eine Beerdigung ist hier zu sehen, keine Aufbahrung, kein Ritual. Der Film zeigt stattdessen Verkehrsströme an einer großen Straßenkreuzung - Autos, Droschken, einen Verkehrspolizisten, »Funktionär des Verkehrs «. ${ }^{39}$ Lange wird diese Einstellung gehalten, länger, als es die unmittelbar narrative Logik begründen würde. Es folgt ein Schnitt auf das dunkel ausgekleidete Innere eines Wagens. Auf der Rückbank, in einer Halbtotalen, sehen wir John und Mary, in ihrem Gesichtsausdruck beide verzweifelt, voneinander abgewandt, zwischen ihnen eine leere Mitte. Daraufhin wieder ein Schnitt, zurück auf die Verkehrskreuzung, in leicht veränderter Kadrierung: drei dunkle Fahrzeuge, die einander folgen, der erste Wagen halb offen, auf der Ladefläche ein Sarg.

Diese in der Deskription kurze Sequenz aus drei Einstellungen dauert im Film trotz ihrer narrativen Ökonomie knapp eine Minute. In einer dreistufigen filmischen Autopsie zeigt sie: außen den unablässig strömenden Verkehr, funktionalistisch, allein der Sachlogik unterworfen; innen die Trauernden, introspektiv gefangen, auf sich selbst zurückgeworfen. Und drittens, in Form einer synthetisierenden Montage: die Gleichzeitigkeit beider Erfahrungen. »Von den Familiendepots der Seele zu den Äußerlichkeiten der Handlung « ${ }^{40}$ ist es allein die filmische Operation der Montage, mittels derer in der hier verhandelten Sequenz eine Wirklichkeit zutage tritt, die durch die eigentümliche Gleichzeitigkeit des Verkehrs geprägt ist; eine Wirklichkeit, von der Béla Balázs glaubte, ihr könne, in der Kultur der Druckerpresse vom Verschwinden bedroht, al-

38 Vgl. Miriam Bratu Hansen: »Ein Massenmedium konstruiert sein Publikum. King Vidors \The Crowd««, in: Inge Münz-Koenen (Hg.), Masse und Medium, Berlin: Akademie 2002, S. 47-59.

39 Kracauer: »Chauffeure grüßen«, S. 376.

40 Lethen: Verhaltenslehren der Kälte, S. 51. 
lein durch den Film zur Sichtbarkeit verholfen werden. ${ }^{41}$ Erst im umfließenden Außen des Verkehrs offenbart sich, was einstmals das Innen der Figuren war - und das Medium des Films scheint wie kein zweites privilegiert, dieser Außenkehrung in einer $»$ Epoche des Simultanen, [...] des Nebeneinander, des Auseinander ${ }^{42}$ Ausdruck zu verleihen. Nicht nur der Unfall der Tochter vollzog sich im sfeindlichen Außen< des Verkehrs, auch die Beerdigung ist durch die Erscheinungsformen der großstädtischen Zirkulation überformt. Aber es ist erst der Film, der aus dem Wahrnehmungsmodell >Verkehr e eine Wahrnehmung macht.

Ein Zwischentitel schließt: »The crowd laughs with you always ... but it will cry with you for only a day«, und erinnert an Plessners Diagnose, der zufolge die »Mitwelt immer unversöhnlich« sei. Dass »nur gespannte Wachsamkeit und die Bereitschaft, sich jederzeit aus Bindungen zu lösen, [...] Mobilität« gewährleistet - hier ist eine solche Maxime Programm. Und »darum darf sich die >vollendete Persona auch >auf keine Eigenschaft festnageln lassen $\ll{ }^{43}{ }^{43}$ Von der völligen Eigenschaftslosigkeit sind die Figuren in King Vidors Film indessen noch weit entfernt. Aber THE CROWD antwortet auf die Frage nach dem >Überleben<, indem anhand seines Protagonisten ein Ablöseprozess verhandelt wird: von der Stase zur Mobilität des Verkehrs, von der individualistischen Ideologie des Bürgertums zum außengeleiteten, auf die Wahrnehmung der Anderen gerichteten, seine Identität in der Fremdwahrnehmung findenden Menschen. David Riesman wird diesen Menschen später einmal den >Radartyp $<$ nennen.

Die Bilder der Leichenwagen, die sich im Verkehr befinden, stellen mehr dar als eine - etwa im Hinblick auf BerLin. DiE Sinfonie DER GROBSTADT - vermeintliche Kuriosität in einem Kabinett voller Schauwerte. Vordergründig der Attraktion von Bewegung verpflichtet, lassen sie sich lesen als ein metonymischer Verweis auf eine avantgardistische Anthropologie der 1920er Jahre. Verkehr ist hier nicht eine Metapher, sondern Paradigma: In den filmischen Bildern des Verkehrs verschränkt sich eine tatsächliche Erfahrung von Wirklichkeit mit einem Entwurf, innerhalb dessen das Feld des Verkehrs als das dominante Modell der Wahrnehmung fungiert.

41 Béla Balázs: Der sichtbare Mensch oder die Kultur des Films, Frankfurt/ Main: Suhrkamp 2001, S. 23.

42 Michel Foucault: »Andere Räume«, in: Karlheinz Barck (Hg.), Aisthesis. Wahrnehmung heute oder Perspektiven einer anderen Ästhetik, Leipzig: Reclam 1990, S. 34-46, hier: S. 34.

43 Lethen: Verhaltenslehren der Kälte, S. 58. 


\section{Versprechen}

In der unverhohlenen Faszination, mit der Ruttmann die dynamisch ausschwingenden Linien der Schienenstränge, die Autokolonnen, Reitertrupps, Droschken und Fußgänger im Verkehrsgewühl abbildet, und in der verheißungsvollen Aussicht auf ein glückendes Leben in der Masse, die Vidors Film skizziert, artikuliert sich ein Versprechen. Indem der Verkehr sachgerechtes Verhalten erzwingt und den empirischen Menschen allein als Verkehrsteilnehmer adressiert, ist er imstande, in Zeiten sozialer Desorganisation ein filmisch evident werdendes Modell abzugeben, mittels dessen sich Verhaltenssicherheit gewährleisten lässt - im Leben und im Überleben. Und das ist ein genuin filmisches Versprechen - eine filmische Imagination. Dass die Figuren dieser Filme unter die Räder gekommen sind, ist ihre Rettung.

An wohl keiner anderen Stelle von THE CROWD wird dies deutlicher als im Motiv des mit einem großen Reklameschild werbenden sandwichman. Ein Motiv, das gleich zweimal bemüht wird - zu Beginn sowie am Ende des Films. Bei seiner ersten Verabredung mit Mary macht sich John über einen solchen sandwichman, der zudem als Clown verkleidetet ist, lustig. Die beiden sitzen auf dem offenen Oberdeck eines Busses und betrachten die Szene (nicht nur aufgrund der räumlichen Anordnung) von oben herab: »The poor sap - I'll bet his father thougt he would be president«, meint John spöttisch - eine Bemerkung, die auf zweierlei verweist: zum einen auf die überheblichen Voraussagungen von Johns eigenem Vater, zum anderen aber auch auf das spätere Schicksal des Helden der, - ikonografisch die Einstellung wiederholend - sich am Ende selbst in der Rolle desjenigen, der im Clownskostüm steckt, inmitten des Verkehrs für einen Schnellimbiss werbend, wiederfinden wird.

Oberflächlich betrachtet ließe sich diese Entwicklung nun dahingehend lesen, dass hier am Ende jemand seine Durchschnittlichkeit zu akzeptieren bereit ist »und sich so als überlebensfähiges Mitglied der Gesellschaft erweist « ${ }^{44}$ Demzufolge hätte John Sims’ Reintegration jedoch einen bitteren Beigeschmack, geschähe sie doch um den Preis einer sozialen Demütigung und im Geiste eines (mehr oder weniger bewussten) Eingeständnisses eigenen Versagens. Tatsächlich wurde THE CROWD immer auch in diese Richtung interpretiert - so spricht Miriam Bratu Hansen etwa von einem »ambivalenten, wenn nicht gar zynischen Akkord «, ${ }^{45}$ mit dem der Film ausklinge, und Hans J. Wulff charakterisiert

44 Bratu Hansen: »Ein Massenmedium konstruiert sein Publikum«, S. 49.

45 Ebd., S. 50. 
John Sims als jemanden, dem am Schluss »die eigene Handlungsohnmacht nun endgültig gewiss« geworden sei, und nennt das Ende $»$ melancholisch «. ${ }^{46}$ Das Sprechen von jemandem, der hier >versagt $\iota$, ist jedoch eine kategorische Sichtweise, die nicht nur an die ungebrochene Wahrnehmung der Figur als »innen-geleiteter« Charakter gebunden ist, sondern selbst einem Standpunkt im Geiste der »Innen-Leitung« entspringt.

John Sims' »seelischer Kreiselkompaß« hat tatsächlich versagt - da treffen sich Sims und Tausende andere, die in den 1920er Jahren diese Erfahrung teilen. Doch scheint der Film nicht auch eine ganz andere Lesart anzubieten? Eine, nach der sich in der Figur John Sims ein Wandel vollzogen hat, der es ihr zum Schluss ermöglicht, die eigene Identität wiederherzustellen, die eben nur als jene eines »innen-geleiteten« Typus »illusorisch von Anfang an« gewesen wäre, wie Bratu Hansen ganz allgemein behauptet? ${ }^{47}$ Was als >Nullpunkt` des Subjektschwundes erscheint, kann auch als Drehpunkt einer Subjektermächtigung gelesen werden. Denn der Film schildert Johns Neuanfang eben nicht als Demütigung. Als zum Clown verkleideter sandwichman ist seine Figur entindividualisiert, Träger einer Rolle, einer Maske, die keine Aufschlüsse mehr über ein Innen zulässt, eigenschaftslos. Mit der Inschrift der von ihm getragenen Werbetafel - »I am always happy because I eat at Schnieder's Grill« - artikuliert sich ein Versprechen, das die Möglichkeiten einer

Identitätskonstruktion verheißt, die nicht mehr an die Zerwürfnisse eines Innen gekoppelt sind. Der Film beschreibt nicht nur die Dekonstruktion eines bürgerlichen Selbstbildes im Verkehr, er schildert auch eine Reintegration, die über die wiedererlangte Erwerbstätigkeit des Protagonisten hinausweist: als Möglichkeit, Identität zu leisten. Oder, im Bilde des Films: zu Hause anzukommen, bei der Familie, in einer Rolle, die zu erfüllen John nunmehr bereit scheint - »für die Erwartungen und Wünsche anderer empfänglich «. ${ }^{48}$ Bei John Belton heißt es einmal: »Vidor's hero is an everyman - a man in and of the crowd. But as an everyman, he is also a no-man - a nobody, whose identity, goals and desires are given to him by others rather than stemming from his own, inner needs. ${ }^{49}$

46 Hans J. Wulff: »The Crowd - Materialien«, in: Medienwissenschaft/Kiel: Berichte und Papiere 3 (1998), S. 23.

47 Bratu Hansen: »Ein Massenmedium konstruiert sein Publikum«, S. 55.

48 Riesman: Die einsame Masse, S. 25.

49 John Belton: American Cinema/American Culture, New York: McGrawHill 1994, S. 128. 
Bei Belton ist das pejorativ gemeint - aber als \Radartyp $\measuredangle$, als Mann ohne Eigenschaften, ist John Sims eine vollendete Persona: ein Verkehrsteilnehmer.

Im Bild des Einzelnen, der urplötzlich Teil einer größeren Zahl ist, artikuliert sich am Ende der 1920er Jahre, so scheint es, eine ferne Sehnsucht nach einer Passung, in der das Subjekt einer Massengesellschaft seine Identität zu verwirklichen vermag. Canetti hat vom »Umschlagen der Berührungsfurcht ${ }^{50}$ gesprochen, die allein die Erfahrung, Teil einer Masse zu sein, verspricht. Diese Sehnsucht bricht sich auch in King Vidors THE CROWD Bahn, der mit einer langen Fahrt zurück, die ein immer größer werdendes Vaudeville-Publikum in den Kader fasst, schließt. Unter den zahllosen Besuchern, als einer unter vielen, der Protagonist, der immer kleiner zu werden scheint, schon bald nicht mehr zu erkennen ist: »Plötzlich ist alles schwarz vor Menschen. $«^{51}$

Herkunft, das ist die filmische Lehre von THE CROWD, ist als soziales Kapital in einer Massengesellschaft nicht nur einem unerhörten Bedeutungsverlust unterlegen. Im Aufgehen in und der Teilhabe am Verkehr artikuliert sich die Utopie, Menschsein zu verwirklichen. >Verkehrsteilnehmer` oder >Radartyp zu sein - das sind symbolische Zaubermittel, die es dem Subjekt gestatten, in der Moderne zu überwintern. Für den Verkehrsteilnehmer werden Städte entworfen, er wird zum `Flaneur〈 und >Passanten<, zu Hause »in der Umgebung der technischen Massenmedien, im zerstreuten Großstadtpublikum «. 52

In der letzten Einstellung von THE CROWD schreibt sich damit auch das Medium Film in selbstreferenzieller Weise ein: Mit dem ins Kader gefassten Vaudeville-Publikum blickt der Film auf die soziale Ordnung, die er hervorbringt - und durch die er selbst hervorgebracht wird. Zwischen Grammophon, Radio und Film ist der neue Typus, den John Sims verkörpert, in seinem Element. Mitte der 1920er Jahre ist der Ausdruck »Zerstreuung $«^{53}$ nicht mehr allein negativ gemeint. »Ablenkung « wird zu einer $»$ Spielart sozialen Verhaltens $« .^{54}$

50 Elias Canetti: Masse und Macht, München: Fischer 1960, S. 12.

51 Ebd.

52 Lethen: Verhaltenslehren der Kälte, S. 237.

53 Siegfried Kracauer: »Das Ornament der Masse«, in: ders., Schriften. Bd. V.2, S. 57-67, hier: S. 57.

54 Walter Benjamin: »Das Kunstwerk im Zeitalter seiner technischen Reproduzierbarkeit«, in: ders., Illuminationen. Ausgewählte Schriften I, Frankfurt/Main: Suhrkamp 1977, S. 136-169, hier S. 175. 
Die hierarchische Welt der Kleinstadt und die massenkulturelle des Konsums bilden am Ende der 1920er Jahre Optionen, die noch durchaus miteinander konkurrieren. Auf dem Weg zu sich selbst liegt für den Menschen der Moderne der gleichermaßen ersehnte wie lustvoll gefürchtete Fiebertraum vom Aufgehen des Einzelnen in der Masse, in Anonymität, Aufenthaltslosigkeit, Zerstreuung und Seinsentlastung. Die Anerkenntnis der Rationalität des Verkehrs allein ist es, die Errettung verspricht.

\section{Literatur}

Anderson, Benedict: Imagined Communities. Reflections of the Origin and Spread of Nationalism, London/New York: Verso 1983.

Asendorf, Christoph: Entgrenzung und Allgegenwart. Die Moderne und das Problem der Distanz, München: Fink 2005.

Balázs, Béla: Der sichtbare Mensch oder die Kultur des Films, Frankfurt/ Main: Suhrkamp 2001.

Baxter, John: King Vidor, New York: Monarch 1976.

Belton, John: American Cinema/American Culture, New York: McGrawHill 1994.

Benjamin, Walter: »Das Kunstwerk im Zeitalter seiner technischen Reproduzierbarkeit«, in: ders., Illuminationen. Ausgewählte Schriften I, Frankfurt/Main: Suhrkamp 1977, S. 136-169.

Bratu Hansen, Miriam: »Ein Massenmedium konstruiert sein Publikum. King Vidors >The Crowd««, in: Inge Münz-Koenen (Hg.), Masse und Medium, Berlin: Akademie 2002, S. 47-59.

Canetti, Elias: Masse und Macht, München: Fischer 1960.

Elias, Norbert: Über den Prozeß der Zivilisation, Frankfurt/Main: Suhrkamp 2001.

Foucault, Michel: »Andere Räume«, in: Karlheinz Barck (Hg.), Aisthesis. Wahrnehmung heute oder Perspektiven einer anderen Ästhetik, Leipzig: Reclam 1990, S. 34-46.

Friedberg, Anne: Window Shopping. Cinema and the Postmodern, Berkeley: University of California Press 1994.

Gömmel, Rainer: »Technik und Mobilität«, in: Armin Hermann/Wilhelm Dettmering (Hg.), Technik und Gesellschaft, Düsseldorf: Georg-Agricula-Gesellschaft 1993, S. 293-313.

Ickstadt, Heinz: »Kommunikationsmüll und Sprachcollage. Die Stadt in der amerikanischen Fiktion der Postmoderne«, in: Scherpe (Hg.), Die Unwirklichkeit der Städte (1988), S. 197-224.

Jünger, Ernst: Das abenteuerliche Herz. Figuren und Capriccios, Stuttgart: Klett Cotta 1979. 
Kracauer, Siegfried: »Chauffeure grüßen«, in: ders., Schriften. Bd. V.1: Aufsätze 1915-1926, Frankfurt/Main: Suhrkamp 1990, S. 376-377.

Kracauer, Siegfried: »Das Ornament der Masse«, in: ders., Schriften, Bd. V.2 (1990), S. 57-67.

Kracauer, Siegfried: Die Angestellten. Aus dem neuesten Deutschland, Frankfurt/Main: Suhrkamp 1971.

Kracauer, Siegfried: »Kleine Signale«, in: ders., Schriften. Bd. V.2 (1990), S. 234-236.

Kracauer, Siegfried: Schriften. Bd. V.2: Aufsätze 1927-1931, Frankfurt/ Main: Suhrkamp 1990.

Kracauer, Siegfried: »Stadt-Erscheinungen«, in: ders., Schriften. Bd. V.3: Aufsätze 1932-1965, Frankfurt/Main: Suhrkamp 1990, S. 90-93.

Lethen, Helmut: Verhaltenslehren der Kälte. Lebensversuche zwischen den Kriegen, Frankfurt/Main: Suhrkamp 1994.

Metz, Christian: »Bemerkungen zu einer Phänomenologie des Narrativen«, in: ders., Semiologie des Films, München: Fink 1972, S. 35-50.

Müller, Lothar: »Die Großstadt als Ort der Moderne«, in: Scherpe (Hg.), Die Unwirklichkeit der Städte (1988), S. 14-33.

Parr, Rolf: ») Gemeinschaft $<$ und 〉Gesellschaft . Diskursive Koordinaten im wilhelminischen Deutschland«, in: ders., Interdiskursive As-Sociation. Studien zu literarisch-kulturellen Gruppierungen zwischen Vormärz und Weimarer Republik, Tübingen: Niemeyer 2000, S. 4754.

Plessner, Helmuth: Grenzen der Gemeinschaft. Eine Kritik des sozialen Radikalismus, Frankfurt/Main: Suhrkamp 2002.

Riesman, David: Die einsame Masse. Eine Untersuchung der Wandlung des amerikanischen Charakters, Reinbek bei Hamburg: Rowohlt 1958.

Roskothen, Johannes: Verkehr. Zu einer poetischen Theorie der Moderne, München: Fink 2003.

Scherpe, Klaus R. (Hg.): Die Unwirklichkeit der Städte. Großstadtdarstellungen zwischen Moderne und Postmoderne, Reinbek: Rowohlt 1988.

Toeplitz, Jerzy: Geschichte des Films. Bd. 1: 1895-1928, Berlin: Henschel 1984.

Vidor, King: A Tree is a Tree. An Autobiography, Hollywood: Samuel French 1981.

Wulff, Hans J.: »The Crowd - Materialien«, in: Medienwissenschaft/ Kiel: Berichte und Papiere 3 (1998). 